\title{
Transmission of Spike Trains at the Retinogeniculate Synapse
}

\author{
Lawrence C. Sincich, Daniel L. Adams, John R. Economides, and Jonathan C. Horton \\ Beckman Vision Center, University of California, San Francisco, San Francisco, California 94143
}

\begin{abstract}
Retinal spikes impinging on relay neurons in the lateral geniculate nucleus (LGN) generate synaptic potentials, which sometimes produce spikes sent to visual cortex. We examined how signal transmission is regulated in the macaque LGN by recording the retinal input to a single LGN neuron while stimulating the receptive field center with a naturalistic luminance sequence. After extracting the EPSPs, which are often partially merged with spike waveforms, we found that $>95 \%$ of spikes were associated with an EPSP from a single retinal ganglion cell. Each spike within a "burst" train was generated by an EPSP, indicating that LGN bursts are inherited from retinal bursts. LGN neurons rarely fired unless at least two EPSPs summated within $40 \mathrm{~ms}$. This facilitation in EPSP efficacy was followed by depression. If a spike was generated by the first EPSP in a pair, it did not alter the efficacy of the second EPSP. Hence, the timing of EPSPs arising from the primary retinal driver governs synaptic efficacy and provides the basis for successful retinogeniculate transmission.
\end{abstract}

Key words: retinal ganglion cells; lateral geniculate nucleus; luminance; natural statistics; spike generation; S-potential; neural coding; T-type calcium current

\section{Introduction}

Ever since the first recordings of connected retinal ganglion cells and lateral geniculate neurons, it has been appreciated that not every retinal spike leads to a geniculate spike (Bishop et al., 1958; Hubel and Wiesel, 1961; Cleland et al., 1971). Efficacy is defined as the probability that a given retinal action potential generates an action potential in a geniculate neuron. It is usually much less than $100 \%$, because many retinal spikes are not conveyed by the lateral geniculate nucleus (LGN) to the primary visual cortex. It is possible, however, for a single retinal spike to generate more than one geniculate spike by triggering a burst. Such events are defined as spikes occurring after a $100 \mathrm{~ms}$ pause, with an interspike interval of $<4$ ms (Sherman, 2001a). During the silent period, the low-threshold $\mathrm{Ca}^{2+}$ current $\left(I_{\mathrm{T}}\right)$ becomes deinactivated. Subsequently, a single retinal EPSP can cause multiple geniculate action potentials riding on a large depolarizing $\mathrm{Ca}^{2+}$ current (Jahnsen and Llinas, 1984; Crunelli et al., 1989; Lu et al., 1992; Zhan et al., 1999; Ramcharan et al., 2000b). This burst would transiently raise efficacy beyond $100 \%$.

To understand LGN function, it is necessary to learn how the probability of transmission varies during burst versus tonic firing. Changes in efficacy have not been examined using natural stimuli or with respect to firing mode. It is not clear what features of the retinal spike train are likely to promote burst firing in the LGN. When a retinal EPSP triggers $I_{\mathrm{T}}$ activation in a geniculate cell, subsequent EPSPs may become immaterial because $I_{\mathrm{T}}$ has

Received Nov. 22, 2006; revised Jan. 13, 2007; accepted Jan. 15, 2007.

This work was supported by National Eye Institute Grants EY10217 (J.C.H.) and EY02162 (Beckman Vision Center). Support was also received from the Larry L. Hillblom Foundation. We thank Ken Park, Amar Marathe, and Cristina Jocson for help with experiments and analysis. Matteo Carandini and Ted Weyand provided valuable comments on this work.

Correspondence should be addressed to Dr. Lawrence C. Sincich, Beckman Vision Center, 10 Koret Way, University of California, San Francisco, San Francisco, CA 94143-0730. E-mail: sincich!@vision.ucsf.edu.

D01:10.1523/JNEUROSCI.5077-06.2007

Copyright $\odot 2007$ Society for Neuroscience $\quad$ 0270-6474/07/272683-10\$15.00/0 driven the membrane potential above threshold. Under these circumstances, the retinal spike train would become effectively desynchronized from the LGN spike train. Another possibility is that certain stimuli may induce burst firing in retinal ganglion cells, and these spikes account for some geniculate bursts. Here, we explore two issues: (1) what happens to efficacy when an LGN neuron fires in burst versus tonic mode during natural stimulation, and (2) how faithfully do LGN spike trains represent retinal spike trains? These questions were addressed by recording extracellularly the action potentials and retinal EPSPs ("S-potentials") of single LGN neurons in the macaque.

\section{Materials and Methods}

Experimental animals and surgical procedures. Experiments were conducted in six rhesus monkeys using procedures approved by the University of California, San Francisco Institutional Animal Care and Use Committee in accordance with National Institutes of Health guidelines. Anesthesia was induced with ketamine $\mathrm{HCl}(10 \mathrm{mg} / \mathrm{kg}$, i.m.). After endotracheal intubation, animals were ventilated with $1-1.5 \%$ isoflurane in a 1:1 mixture of $\mathrm{N}_{2} \mathrm{O} / \mathrm{O}_{2}$ to maintain anesthesia. We monitored the electrocardiogram, electroencephalogram, respiratory rate, body temperature, blood oxygenation $\left(\mathrm{S}_{\mathrm{p}} \mathrm{O}_{2}\right)$, end-tidal $\mathrm{CO}_{2}$, urine output, and inspired/expired levels of anesthetic gases throughout each $4-5 \mathrm{~d}$ experiment. A $5 \%$ dextrose in $0.45 \%$ saline solution was given intravenously at $3 \mathrm{ml} \cdot \mathrm{kg}^{-1} \cdot \mathrm{h}^{-1}$. After the animal was placed in a stereotaxic frame, we made a small craniotomy at a location suitable for dorsal access to the LGN (7-17 mm mediolaterally, 2-12 $\mathrm{mm}$ anteroposteriorly). Before recording, neuromuscular blockade was established with vecuronium bromide $\left(60 \mu \mathrm{g} \cdot \mathrm{kg}^{-1} \cdot \mathrm{h}^{-1}\right)$ to prevent eye movements. We blocked lens accommodation with $1 \%$ cyclopentolate hydrochloride (Cyclogyl; Alcon Laboratories, Fort Worth, TX) and installed contact lenses to focus the eyes on a translucent tangent screen $144 \mathrm{~cm}$ away. Clarity of the optics was routinely checked and maintained throughout the course of the recordings.

Electrophysiological recording and visual stimulation. A single Epoxylite-coated tungsten electrode (5-8 M $\Omega$; Frederick Haer Company, Bowdoinham, ME) was lowered through a stainless steel guide tube positioned $5 \mathrm{~mm}$ above the LGN. Extracellular potentials were recorded 
with $1000 \times$ differential amplification (model 1800; A-M Systems, Carlsborg, WA), with the guide tube serving as reference electrode. Analog potentials were bandpass filtered between 0.3 and $3 \mathrm{kHz}$ (occasionally between 0.001 and $3 \mathrm{kHz}$ to verify the temporal profile of EPSPs during recordings) before being acquired digitally at $25 \mathrm{kHz}$ (Power 1401; Cambridge Electronic Design, Cambridge, UK). We made electrolytic lesions to verify recording sites by passing $\pm 160 \mu \mathrm{A}$ of square-wave alternating current at $16 \mathrm{kHz}$ for $30 \mathrm{~s}$ (model STG 1001; Multi Channel Systems, Reutlingen, Germany).

Once isolated somatic action potentials were encountered, it was useful to advance the electrode slowly in $2-4 \mu \mathrm{m}$ increments to find the retinal EPSPs associated with the geniculate neurons. The evolution of an LGN spike and the appearance of its EPSP during the approach to one neuron is illustrated in Figure $1 B$. When an LGN spike with an EPSP amplitude $>0.05 \mathrm{mV}$ was isolated, we plotted the boundaries of the receptive field center with a hand lamp.

For quantitative data collection, visual stimuli were delivered by directing the beam of a diffused light-emitting diode (LED) toward the back of a translucent screen at the center of the receptive field. To prevent stimulation of the receptive field surround a black-flocked foil mask with a circular hole the size of the receptive field center was placed on the front of the screen. We specifically restricted the stimulus to the receptive field center to minimize any stimulation of extraneous retinal ganglion cells, whose field centers may have overlapped the surround of the cell being recorded.

We designed the visual stimulus to vary in brightness over time at 80 $\mathrm{Hz}$, with a temporal power spectrum that approximated the luminance intensities measured when viewing natural scenes (van Hateren, 1997; Reinagel and Reid, 2000). We refer to this stimulus as "naturalistic" to underscore the fact that van Hateren (1997) did not incorporate normal eye movements in acquiring temporal luminance data. We generated a series of unique $5 \mathrm{~s}$ sequences of luminance changes, having a mean power spectrum that followed a $1 / f_{t}^{\gamma}$ distribution, with $f_{t}$ being the temporal frequency, and $\gamma=0.7$ (supplemental Fig. 1, available at www.jneurosci.org as supplemental material). The power spectrum was weighted toward temporal frequencies $<10 \mathrm{~Hz}$, a range that drives LGN neurons well and potentially favors burst firing activity (Derrington and Lennie, 1984). To ascertain the reliability of firing to repeated stimuli between each unique $5 \mathrm{~s}$ sequence, we interleaved one identical $5 \mathrm{~s}$ sequence with the same power spectrum as the unique sequences. The value of $\gamma$ was somewhat low compared with measured statistics because our sequences were temporally clipped at $0.2 \mathrm{~Hz}$ to allow presentation of enough repeated sequences and at $80 \mathrm{~Hz}$ because LGN neurons are not responsive above this frequency (Derrington and Lennie, 1984). Except when noted, all spike analyses were based on responses to the unique stimuli.

For each neuron, we selected the LED color and mask aperture diameter that yielded the briskest response, as judged by audio monitoring of the firing rate to a $3 \mathrm{~Hz}$ on-off stimulus. The peak LED wavelengths used were red $(660 \mathrm{~nm})$, yellow $(592 \mathrm{~nm})$, green $(502 \mathrm{~nm})$, blue $(467 \mathrm{~nm})$, or white (nominally $550 \mathrm{~nm}$ ). Linearity of the LED intensity was achieved by driving it through a circuit that converted a linear command voltage into a frequency-modulated pulse train, with each $1.6 \mu$ s square-wave pulse having a constant voltage amplitude (Swanson et al., 1987). We confirmed the linearity of the stimulus with a Minolta LS-100 luminance meter. The luminance values were varied between 256 equally spaced levels, and we adjusted the mean luminance value for each neuron such that firing rates were nearly saturated during the $3 \mathrm{~Hz}$ on-off stimulus.

To classify the neurons functionally, we compiled contrast and temporal frequency tuning curves. For the contrast stimuli, the LED luminance was stepped, at $4 \mathrm{~Hz}$, through a series of luminance values, from a dark baseline (to stimulate ON cells) or from a bright baseline (to stimulate OFF cells). The temporal frequency stimulus consisted of a fullcontrast sine wave that swept from 0.2 to $96 \mathrm{~Hz}$ and back. LGN neurons that responded at low contrast and high frequency were classified as magnocellular, whereas neurons with high contrast requirements and low temporal frequency tuning were classified as parvocellular (Derrington and Lennie, 1984; Levitt et al., 2001). We were uncertain whether any koniocellular neurons were recorded because we did not rigorously

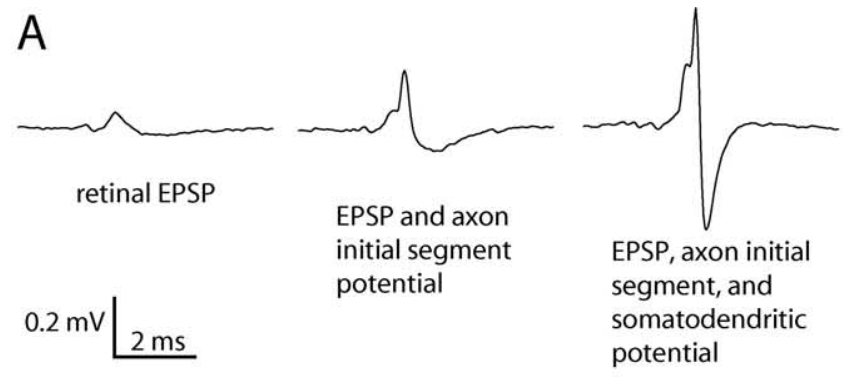

B

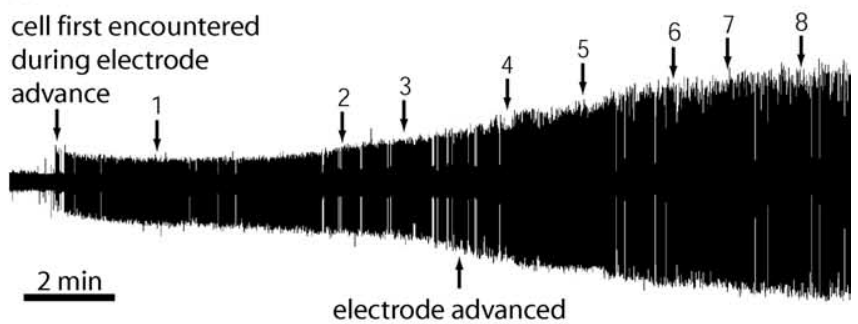

$50 \mu \mathrm{m}$ since start

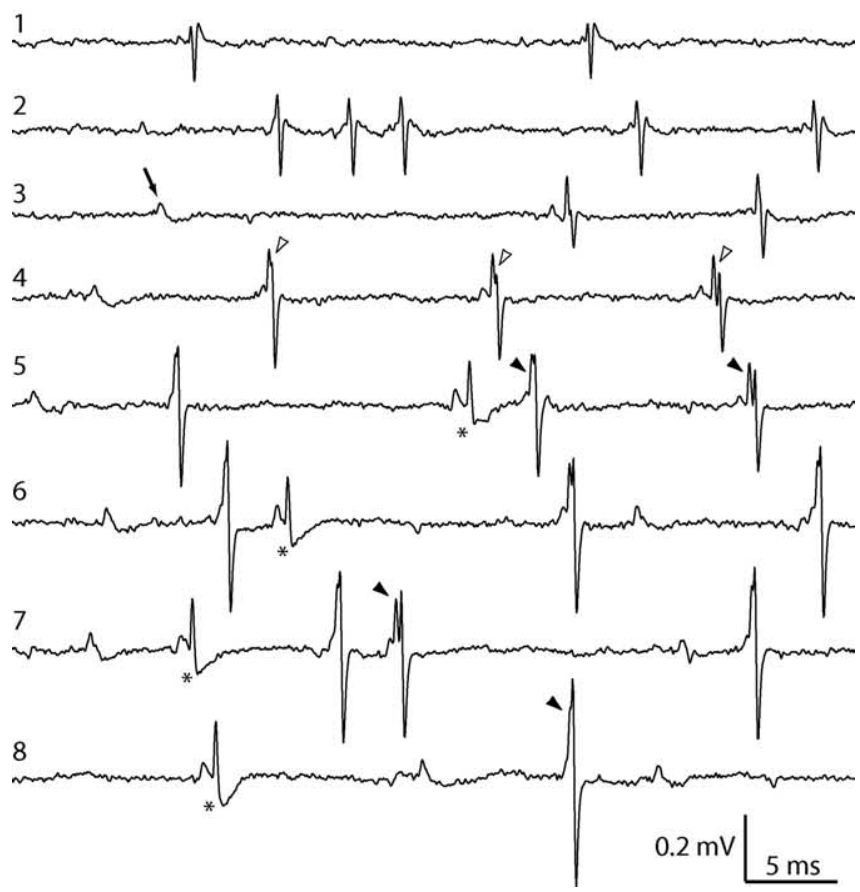

Figure 1. Variation in LGN spike shapes and appearance of retinal EPSPs as a microelectrode approaches a single LGN neuron. $A$, Examples of the three typical waveforms: isolated EPSP, EPSP with axon initial segment potential, and EPSP with axon initial segment plus somatodendritic potential. $\boldsymbol{B}$, During this $15 \mathrm{~min}$ record of spontaneous activity, the electrode was advanced $80 \mu \mathrm{m}$ from the point at which the LGN cell was first detected. The initial waveform (at time point 1) was triphasic, characteristic of an LGN spike recorded at a distance. By time point 3 , retinal EPSPs began to appear in isolation (arrow) and immediately preceding LGN spikes. At time point 4 , after a $50 \mu \mathrm{m}$ electrode advance, the two potentials in the $L G N$ waveform became discernable, signified by a notch between the axon initial segment potential and the somatodendritic potential (open arrowheads). The combined waveform varies in shape because of the slight temporal jitter that separates the action potential initiated at the axon initial segment from its propagation back into the somatodendritic compartment (filled arrowheads; time points 5-8). Axon initial segment potentials occasionally failed to invade the soma and dendrites (asterisks). Voltage scale applies to all traces.

test for color selectivity. The same classification scheme was used to distinguish parasol and midget retinal ganglion cells for recordings made in the optic tract. Recording sites in the LGN were determined by histological examination of the electrode tracks and lesions. For 11 of the 15 
cells analyzed, a definitive classification could be made based on physiological properties and the location of the recording site verified histologically (supplemental Table 1, available at www.jneurosci.org as supplemental material).

Data analysis. Retinal EPSPs and geniculate action potentials were identified by off-line waveform templating (Spike2; Cambridge Electronics Design). In general, we observed three potentials: isolated EPSPs (S-potentials), monophasic axon initial segment potentials, and biphasic somatodendritic potentials. All three types have been well characterized previously in the cat and macaque LGN and are recognized to be different electrical events in the same cell (Hubel and Wiesel, 1961; Bishop et al., 1962; Lee et al., 1983; Kaplan and Shapley, 1984; Wang et al., 1985). Using templates of 1.5-2 ms duration, we found that the potentials occurred as three waveforms with different relative amplitudes. Typically, isolated EPSPs were 0.05-0.2 mV, EPSPs with axon initial segment potentials were $0.2-0.4 \mathrm{mV}$, and EPSPs combined with axon initial segment and somatodendritic potentials ranged from 0.5 to $2 \mathrm{mV}$ peak-to-trough (Fig. 1A).

EPSPs were never observed unless the electrode was very close to a cell body, picking up an action potential with a signal-to-noise ratio of at least 20:1. Action potentials recorded extracellularly had a consistently narrow half-width $(<0.2 \mathrm{~ms})$, which was generally independent of distance from the electrode. It has been shown in vitro that action potentials initiate near the soma and invade the dendrites after a short delay (Williams and Stuart, 2000). Occasionally, this invasion fails, resulting in a lone axon initial segment potential. When invasion occurs, temporal jitter between axon initial segment and somatodendritic potentials results in a complex, variable waveform with a notch or inflection in its positive phase, depending on the length of the delay (Bishop et al., 1958; Hubel and Wiesel, 1961). Although this complex waveform and the axon initial segment potential vary in morphology, they both represent a single spike sent to cortex. Therefore, either type of event was treated as a single LGN spike, unless otherwise specified. Isolated somatodendritic potentials were never observed without an immediately preceding initial segment potential.

We classified retinal EPSPs and LGN spikes into three categories according to interspike interval (ISI) (Alitto et al., 2005). Burst spikes were defined as those in a train with an ISI $<4 \mathrm{~ms}$, with the added condition that the train had to be preceded by a silent period $\geq 100 \mathrm{~ms}$. This timing criteria for burst spikes has been shown to reflect the dynamics of the thalamic $I_{\mathrm{T}}$ current (Lu et al., 1992). A short-ISI spike was any spike in a train with an ISI $<4 \mathrm{~ms}$, but the pretrain silent interval was $<100 \mathrm{~ms}$. All remaining spikes were designated as tonic.

It was important to identify unambiguously the EPSPs that evoked action potentials. The waveforms of EPSPs and action potentials were usually partially superimposed, except when the latency between the EPSP and LGN spike was longer than $1 \mathrm{~ms}$. Waveform subtraction can identify overlapping potentials when they are significantly above the noise level (Lewicki, 1998). The basic idea is to create a template waveform of an LGN spike known to lack a merged EPSP and then subtract this template from other spikes in the population, creating residual waveforms caused by the EPSPs. The resulting residual waveforms can be compared with the average isolated EPSP waveform. Because spike shape varied for each neuron, the template was made on a cell-by-cell basis.

For the subtraction procedure, all spikes in the record were extracted in $4 \mathrm{~ms}$ segments and cubic spline interpolated to yield an effective data sample rate of $100 \mathrm{kHz}$. To find a template LGN spike, we searched the record for instances in which the latency between an EPSP and a spike was longer than $1 \mathrm{~ms}$ but shorter than the absolute refractory period of the EPSP. These criteria guaranteed that there was no EPSP merged with the axon initial segment potential in the template. We aligned the template at the half-height point of the axon initial segment potential of each LGN spike (usually $0.2-0.3 \mathrm{mV}$ ) and subtracted the two waveforms. The residual waveforms contained any voltage fluctuation not attributable to an axon initial segment spike. It is noteworthy that some voltage nonlinearity occurred when the EPSP and spike waveforms were partially merged, because the subtracted waveforms were not perfect replicas of isolated EPSPs.

Histology. At the end of an experiment, the animal was given a lethal
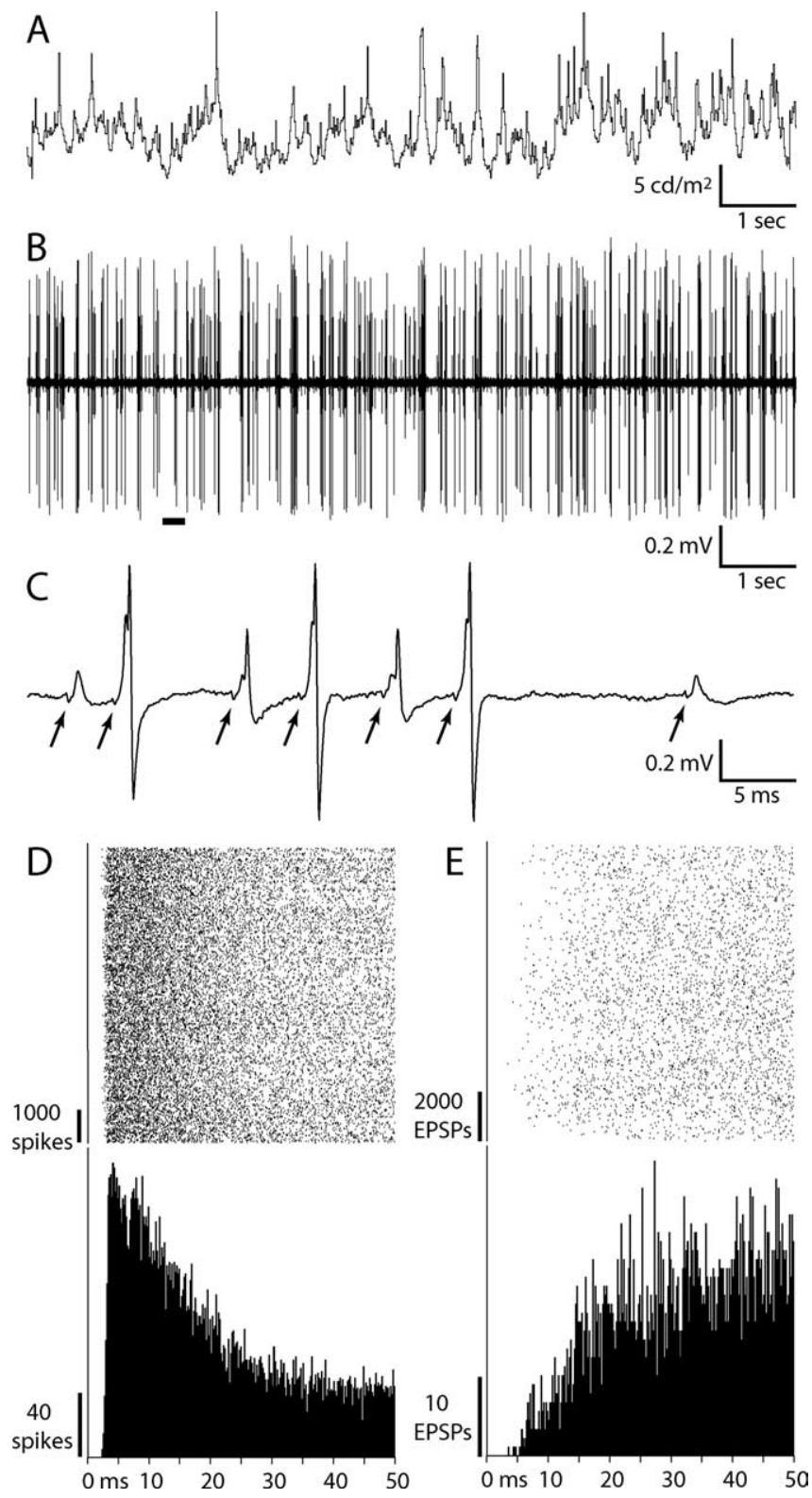

Figure 2. LGN action potentials and EPSPs recorded during visual stimulation. $\boldsymbol{A}$, Luminance modulation of $1 / 2^{\circ}$ spot of light centered on the receptive field of an ON magnocellular neuron (mean luminance, $6.1 \mathrm{~cd} / \mathrm{m}^{2}$ ). $\boldsymbol{B}$, Raw electrode trace acquired during this $10 \mathrm{~s}$ stimulus epoch. $\boldsymbol{C}$, Expanded trace of the underlined portion in $\boldsymbol{B}$, illustrating the occurrence of retinal EPSPS associated with LGN spikes. EPSPs that occur at the beginning of the largest spikes are often difficult to identify because the waveforms are partially merged. The miniscule deflection ("Tpotential") in front of each EPSP (arrows) arises from the retinal action potential invading the axon terminals (Wang et al., 1985), providing independent evidence that an EPSP precedes each LGN spike. $D$, Spike rasters aligned on each axon initial segment potential (top) and resulting interspike interval histogram (bottom; $n=9044$ ). This $L G N$ neuron had an absolute refractory period of $2.5 \mathrm{~ms}$. $\boldsymbol{E}$, Rasters and interval histogram for isolated EPSPs, not associated with LGN spikes, compiled over the same time period as in $\boldsymbol{D}(n=11,873)$. The isolated EPSPs had a refractory period of $3.5 \mathrm{~ms}$.

dose of pentobarbital $(150 \mathrm{mg} / \mathrm{kg})$ and perfused with $2 \mathrm{~L}$ of $0.9 \%$ saline followed by $1 \mathrm{~L}$ of $1 \%$ paraformaldehyde in $0.1 \mathrm{~m}$ phosphate buffer. We removed the brain and blocked out the thalamus. The tissue block was kept in $1 \%$ paraformaldehyde plus $30 \%$ sucrose in phosphate buffer until it sank. The tissue was cut frozen with a microtome into $80 \mu \mathrm{m}$ coronal sections, mounted, and dried on gelatin-coated slides. We processed alternate sections for Nissl substance or cytochrome oxidase histochemistry. 


\section{Results}

Identification of retinal input to LGN neurons

From 240 extracellular electrode recordings made in the LGN of six macaques, we recorded 37 neurons with associated retinal EPSPs, recognizable as smaller, slower potentials that precede the larger LGN action potentials (Lee et al., 1983; Kaplan and Shapley, 1984; Wang et al., 1985). Figure 1 shows a typical recording of the change in spike shape and the appearance of the EPSP as the electrode approached the LGN neuron. An EPSP always became detectable once an LGN spike amplitude exceeded $0.5 \mathrm{mV}$. This finding implies that units with recordable EPSPs are not a special subset of LGN cells but simply those recorded by an electrode located extremely close to the soma. From the population of 37 neurons, we acquired sufficient data for quantitative analysis from 15 cells.

A $10 \mathrm{~s}$ segment of the stimulus and the resulting raw electrode trace from one cell is shown in Figure 2, $A$ and $B$. Retinal EPSPs, axon initial segment potentials, and somatodendritic potentials were all unambiguous (Fig. 2C). The interspike intervals for LGN spikes and isolated EPSPs revealed absolute refractory periods (Fig. $2 D, E)$, indicating that each type of event arose from a single neuron. In 3 of the 37 LGN cells, we recorded EPSPs that lacked an absolute refractory period, indicating that they arose from multiple retinal ganglion cells (supplemental Fig. 2, available at www.jneurosci.org as supplemental material). These cells were not analyzed further.

For this study, it was necessary to determine what proportion of LGN spikes had an accompanying EPSP. The difficulty was that EPSPs were often merged with the rising slope of LGN action potentials. We addressed this problem by extracting EPSP waveforms through a subtraction procedure (Fig. $3 A$ ). Subtracting a template spike from a large sample of LGN spikes yielded a family of residual waveforms that exhibited a smooth but narrow continuum of delays between EPSPs and LGN spikes. The traces were ordered by the time of their peaks (Fig. $3 B, C$ ). For all of the subtracted waveforms of each cell in Figure 3, the EPSP distributions showed two populations of delays before a spike (Fig. 4). The majority of the EPSPs preceded action potentials by $<0.5 \mathrm{~ms}$; consequently, they were partially merged with the LGN spike. A much smaller population had delays that were longer than the refractory period of the retinal cell. For those subtracted traces that had only one EPSP and a delay longer than the refractory period, it was not possible to determine whether the EPSP actually caused the LGN spike.

The subtraction procedure facilitates analysis by making merged EPSPs more obvious. It is important to emphasize that EPSPs are also recognizable on the original spikes, having a char-

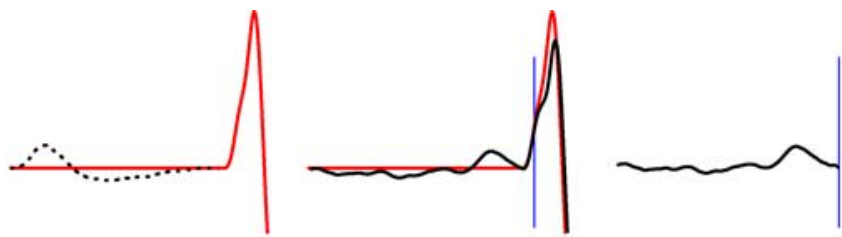

Align template with each spike Subtract template from Create template spike without EPSP at the mid-rise point of axon initial segment potential
Subtract template from
each spike to reveal EPSF

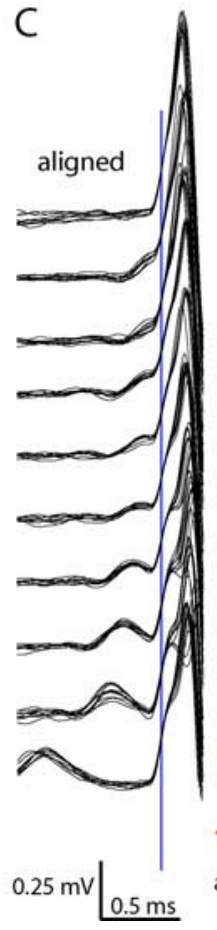

Magno ON cell

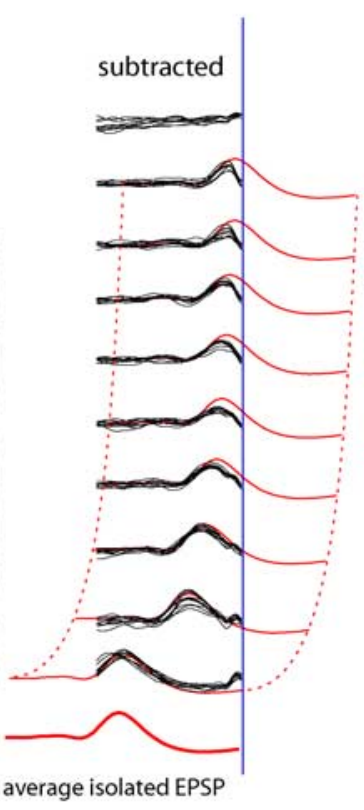

Figure 3. Identification of retinal EPSPs associated with LGN spikes. $\boldsymbol{A}$, Subtraction of an LGN spike template. First, an LGN spike is selected with a latency long enough to separate it completely from the EPSP waveform but short enough to fall within the potential. Next, each LGN spike is aligned at the mid-rise point of the axon initial segment potential (blue line). Finally, the LGN

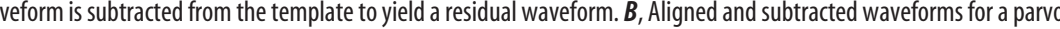
equal intervals along the distribution of EPSP-LGN spike latencies to show the range. The subtracted waveforms are superimposed on the average isolated EPSP (in red) to illustrate how the part of each EPSP that has not merged with the action potential resembles the analogous part of the average EPSP. C, Same analysis as in $\boldsymbol{B}$, for a magnocellular $0 \mathrm{~N}$ cell.

acteristically shallow slope that produces the inflection on the rising phase of the axon initial segment potential (Fig. $3 B, C$, aligned). Note that the rising slope of the residual waveforms is similar to the rising slope of the average isolated EPSP. This similarity is made more apparent by superimposing the subtracted traces onto the average EPSP (Fig. $3 B, C$, subtracted).

Remarkably, for our population of LGN neurons, extremely few spikes lacked an EPSP within the preceding 2 ms. Of 11,751 spikes analyzed from the parvocellular neuron in Figure $3 B, \sim 5 \%$ of the subtracted waveforms were virtually flat. For the magnocellular neuron, $<1 \%$ of the 5545 subtracted waveforms analyzed showed no evidence of an EPSP (Fig. 3C). Analysis of the other 13 cells in our population revealed that between 94.6 and $99.7 \%$ of the LGN spikes were driven by retinal EPSPs falling within the refractory period of each cell. These data indicate that, under our stimulus conditions, macaque LGN neurons rarely fired without an identifiable EPSP from a retinal ganglion cell. 


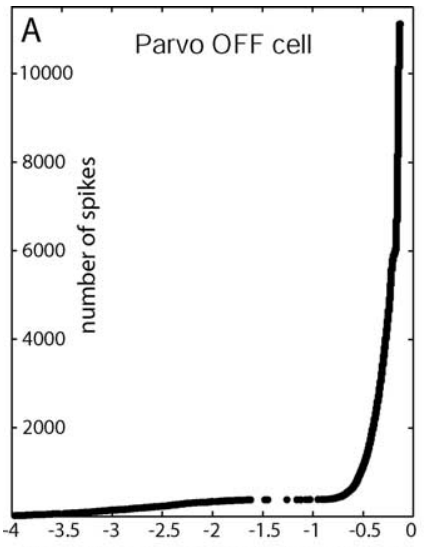

Time of peak in subtracted traces (ms)

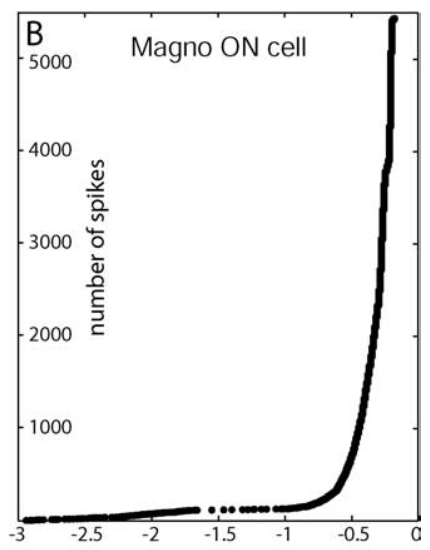

Time of peak in subtracted traces (ms)
Figure 4. Distribution of latencies between subtracted EPSP waveform peaks and LGN spikes. $\boldsymbol{A}$, For the cell in Figure $3 B$, the time of the peaks in the subtracted waveforms are plotted, in order of latency, with respect to the peak of the axon initial segment potential. The axon initial segment potential was designated $t=0$ because the somatodendritic spike often failed in this cell. The gap in the distribution near $-1.5 \mathrm{~ms}$ is attributable to the refractory period of the EPSP. $\boldsymbol{B}$, Distribution of latencies plotted for the cell in Figure $3 C$. The latencies are shifted in this cell because they are plotted with respect to the peak of the somatodendritic potentials, which have a longer delay after EPSPs than the axon initial segment potentials. Parvo, Parvocellular; Magno, magnocellular.

\section{Retinal activity during burst and tonic modes in the LGN}

We next examined whether the likelihood of a retinal EPSP driving an LGN spike depended on the firing mode of the LGN neuron. Thalamic neurons are known to generate a burst of action potentials in response to activation of the calcium current, $I_{\mathrm{T}}$, which occurs after prolonged hyperpolarization of the membrane potential (Jahnsen and Llinas, 1984; Crunelli et al., 1989; Lu et al., 1992; Zhan et al., 1999). These spikes require only a single depolarizing event to occur because $I_{\mathrm{T}}$ keeps the membrane above threshold for tens of milliseconds before inactivating. The few nonretinally driven spikes in our population may have been such $I_{\mathrm{T}}$-driven events.

We first classified spikes into three categories according to interspike intervals: "burst" (spikes with an ISI $<4 \mathrm{~ms}$ that followed a $100 \mathrm{~ms}$ silent period), "short-ISI" (4 ms ISIs without the preliminary silence), and "tonic" (all remaining spikes). A scatter plot of the prespike versus postspike time intervals of the parvocellular neuron analyzed in Figure $3 B$ illustrates the prevalence of the spikes in each category (supplemental Fig. 3, available at www.jneurosci.org as supplemental material). Although our stimulus had a power spectrum with most of its energy in the low temporal frequencies, potentially favoring burst firing, the interspike times exhibited a wide range of intervals dominated by tonic firing. For this cell, $90 \%$ of the spikes were tonic, 5.7\% were short-ISI, and $4.3 \%$ were burst. Comparable proportions of spike types were found across our population of LGN cells (tonic, $86.6 \pm 14.8 \%$; short-ISI, $9.6 \pm 10.5 \%$; burst, $3.7 \pm 4.6 \%$ ) (supplemental Table 1, available at www.jneurosci.org as supplemental material). The majority of burst trains consisted of two spikes. The prevalence of burst firing matched the range reported previously in the macaque during visual stimulation with a $5 \mathrm{~Hz}$ grating (Ramcharan et al., 2000a).

How LGN neurons handle retinal input during bursts is unknown. If $I_{\mathrm{T}}$ were activated during naturalistic stimulation, the membrane voltage would transiently exceed threshold and generate multiple spikes. LGN spikes might therefore become temporarily dissociated from retinal input during stimuli that cause bursts. A selection of raw traces containing bursts from a parvocellular neuron illustrates that this scenario did not occur (Fig. $5 A)$. Nearly every LGN spike in a burst, not just the first spike, had an EPSP. We applied the subtraction procedure to secondary spikes in the bursts and compared their EPSP-to-spike latency distribution with that of the whole population (Fig. 5B). Most secondary burst spikes had latencies $<0.5 \mathrm{~ms}$, just like the latencies for other spike types. The distribution was biased toward shorter times compared with the overall population of EPSPs, an effect expected because bursts have an inherently high firing rate. We also examined the latency distribution of secondary short-ISI spikes. In most cases, these spikes also had shorter latencies compared with tonic spikes. As we will see in the next section, when an LGN neuron is driven at a high rate, the incoming EPSPs are more quickly transduced. This presumably occurs because the membrane potential is very near threshold. The presence of identifiable EPSPs on virtually all secondary and higher-order burst spikes means that the number of spikes in a burst is determined by retinal input. Given the dynamics of $I_{\mathrm{T}}$ current, this result suggests that $I_{\mathrm{T}}$ may not have been activated during these bursts, but definitive proof would require intracellular recording.

These data show that bursts in the LGN are driven by bursts in the retina. It has not been reported that retinal ganglion cells can fire in bursts during naturalistic stimulation. We therefore recorded 13 units in the optic tract of two macaques. Stimuli that elicited bursts in LGN neurons also did so in retinal ganglion cells. Moreover, retinal firing patterns were similar to EPSP trains recorded in LGN neurons (Fig. 5C). This finding suggests that retinal bursts identified by EPSPs faithfully represent the activity of retinal ganglion cells, as recorded directly from the optic tract. Retinal ganglion cells were categorized as midget $(n=8)$ or parasol $(n=5)$ based on their contrast and temporal frequency tuning properties. Cells in both classes showed burst activity. However, retinal bursts were uncommon. Their prevalence equaled that of LGN neurons (retinal bursts, $3.5 \pm 2.1 \%$; LGN bursts, $3.7 \pm 4.6 \%$ ). The mean firing rates in the optic tract were comparable with mean rates for EPSPs, providing inferential evidence that few EPSPs were missed when recording in the LGN (EPSPs, $28.7 \pm 9.3$ events/s; tract fibers, $31.2 \pm 11.6$ spikes/s). We conclude that the LGN firing patterns are very tightly coupled to retinal EPSPs under our stimulus conditions, regardless of firing mode.

\section{EPSP efficacy and summation requirement of LGN action potentials}

Previous studies of connected retinal ganglion cells and LGN neurons have shown that mean firing rates are lower in LGN neurons (Kaplan et al., 1987; Movshon et al., 2005). In our population of 15 cells, the average probability of a retinal ganglion cell spike leading to an LGN spike was $46 \pm 16 \%$. This overall efficacy rate does not convey how the probability of transmission varies during visual stimulation or how it depends on the recent history of spiking events. With the opportunity to record the input and output spike trains of a single pair of neurons, we asked how the efficacy of an input is modulated in time and how the output depends on preceding EPSPs. Figure $6 \mathrm{~A}$ compares the perispike time correlograms of LGN spikes and EPSPs, generated for each spike category from a series of rasters. The auto-correlogram for the LGN spike (shown in black) is centered at $t=0$ for each tonic LGN spike or on the first spike in each short-ISI or burst train (Fig. 6B). For this cell, the auto-correlogram shows that LGN spiking generally occurred in clusters during naturalistic stimulation, as in the cat (Dan et al., 1996). The LGN firing rate was 
elevated for a period of $\pm 25 \mathrm{~ms}$ around the tonic and short-ISI LGN spikes compared with the mean firing rate. Elevated firing was also observed for $30 \mathrm{~ms}$ after LGN burst trains. Similarly, the LGNEPSP cross-correlograms (Fig. $6 B$, shown in red) demonstrate that retinal firing was more frequent $\pm 30 \mathrm{~ms}$ around an LGN spike for all spike categories and fell below the mean at longer times. For burst spikes in particular, a large number of failed EPSPs occurred just before bursts. In fact, for the 10,591 bursts recorded in our 15 cells, there was at least one failed EPSP before all but one burst. The rate of these failed EPSPs peaked at $4 \mathrm{~ms}$ before bursts (Fig. 6B).

These data show that clustered LGN firing originates from clustered retinal input, but the LGN firing pattern was not simply the result of a constant rate of successful EPSP transmission. Instead, the success rate changed on a millisecond timescale during visual stimulation. Dividing the auto-correlogram by the crosscorrelogram for each cell yields an index of efficacy before and after a tonic, short-ISI, or burst LGN spike (Fig. 6C). These population EPSP efficacy profiles depict the average likelihood of successful transmission in the period surrounding each spike during naturalistic stimulation. For all spike classes, EPSP efficacy after a spike peaked at $\sim 70 \%$, well above the mean efficacy rate of $46 \%$. On average, this facilitation lasted $\sim 40 \mathrm{~ms}$. Beyond $40 \mathrm{~ms}$, mean efficacy was depressed for tonic and burst spikes. This result indicates the presence of two mechanisms: short-term pairedspike facilitation followed by lingering paired-spike suppression. An increase in efficacy occurred before short-ISI trains, indicating that short-ISI trains tend to occur in the context of above average firing rates. However, shortISI trains were not followed by a drop in efficacy.

The increase in efficacy of an EPSP observed shortly after an LGN spike is consistent with a previous report of retinal pairedspike facilitation (Usrey et al., 1998). When EPSPs arrive in rapid succession, does the efficacy of the second EPSP depend on whether the first EPSP generates a spike (Fig. 7A)? To address this issue, we compared the efficacy of the second EPSP in a pair of EPSPs, contingent on whether the first EPSP was successfully transmitted (as occurred in $46 \%$ of EPSP pairs). For the cell analyzed in Figure 6, plots of second EPSP efficacy after conduction versus failure of the first EPSP had similar profiles, although efficacy after an LGN spike began slightly higher and fell off more quickly (Fig. $7 B$ ). Across the population of cells, there was no significant difference in mean second EPSP efficacy after a conducted versus failed first EPSP at any ISI (Fig. 7C) ( $t$ test, $p>0.1$ for all ISIs between 3 and $40 \mathrm{~ms}$ ). Note that, for ISIs longer than $40 \mathrm{~ms}$, efficacy fell to nearly 0. LGN spikes virtually never occurred after such long ISIs, providing evidence that neurons needed to be "primed" within $40 \mathrm{~ms}$ by an EPSP to fire.
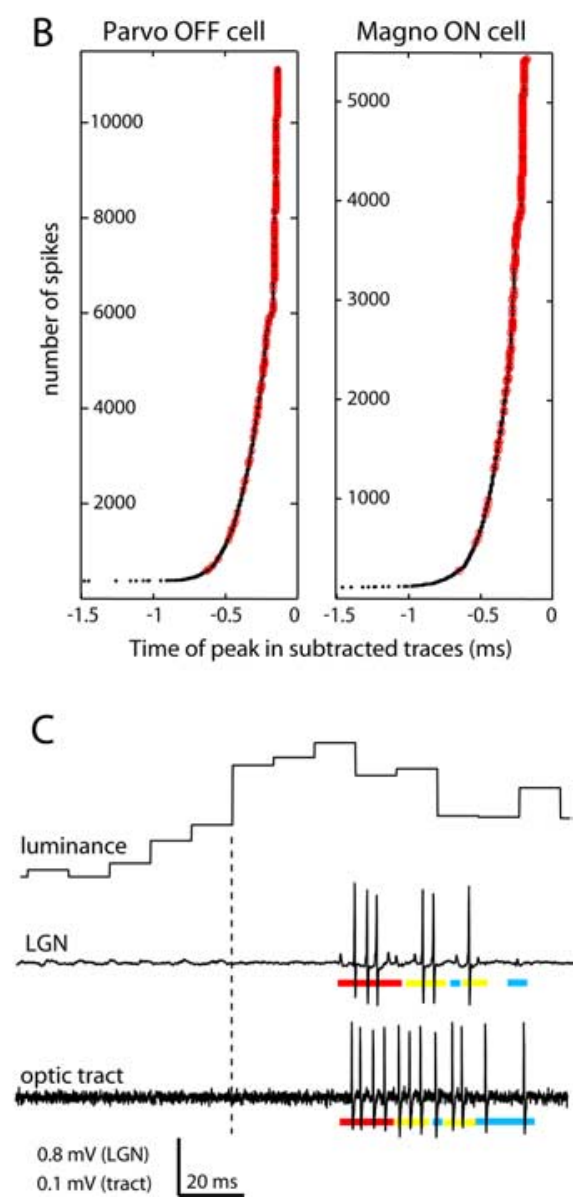

Figure 5. Retinal EPSPs drive nearly all LGN spikes during bursts. $A$, A representative sample of burst spike trains from the cell in Figure 3B, illustrating the range of EPSP-to-spike latencies. Each of these trains was preceded by $100 \mathrm{~ms}$ with no LGN spikes. Red mark spikes with identifiable EPSPs within $0.5 \mathrm{~ms}$ of the axon initial segment potential. Broken gray line shows that the amplitudes of isolated and merged EPSPs are similar. B, Latencies between subtracted EPSP waveforms and all spikes (black dots, 作 Although these recordings were made in different animals, the retinal burst trains were similar whether recorded as optic tract spikes or as EPSPs in the LGN. Parvo, Parvocellular; Magno, magnocellular.

This analysis treated EPSP pairs without regard for preceding EPSPs. Conceivably, the enhanced efficacy after an LGN spike is actually attributable to an EPSP that occurred before the first EPSP in a pair. Therefore, we compared efficacies when the failed EPSP ISI equaled the successful EPSP ISI plus the interval since the previous EPSP (supplemental Fig. 4, available at www. jneurosci.org as supplemental material). This control analysis showed that the enhanced efficacy after a spike cannot be explained by the presence of an EPSP preceding any pair of EPSPs. We conclude that the influence of an EPSP on subsequent EPSP efficacy does not depend on whether the first EPSP is transmitted, suggesting that the spike-generating mechanism does not interfere with EPSP summation.

We next considered the influence of an EPSP that preceded any pair in more detail. Efficacy was examined as a function of the ISI of the paired EPSPs and the time since the preceding EPSP ("dead time"). This analysis provided a comprehensive picture of how multiple EPSPs integrate in time. For second EPSPs, dead time affected efficacy when ISIs were $<30 \mathrm{~ms}$ but had no effect at longer ISIs (Fig. 7D). For ISIs shorter than $30 \mathrm{~ms}$, all three EPSPs 
A
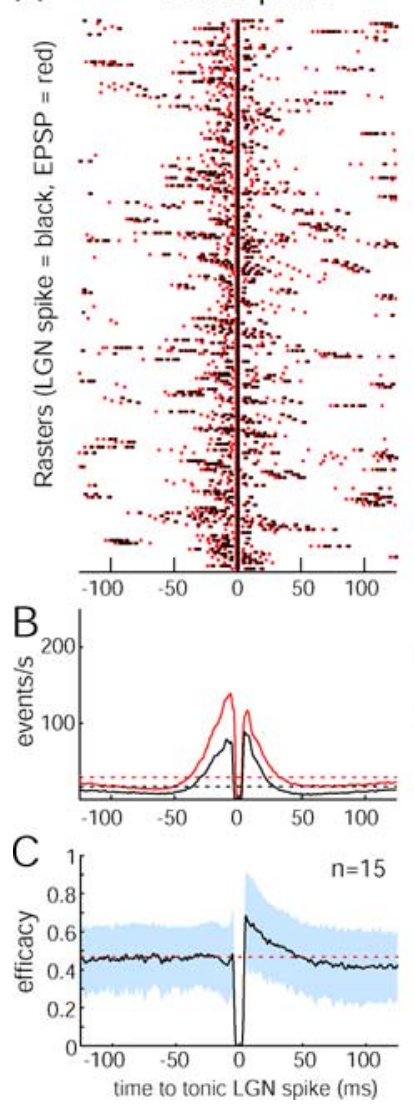
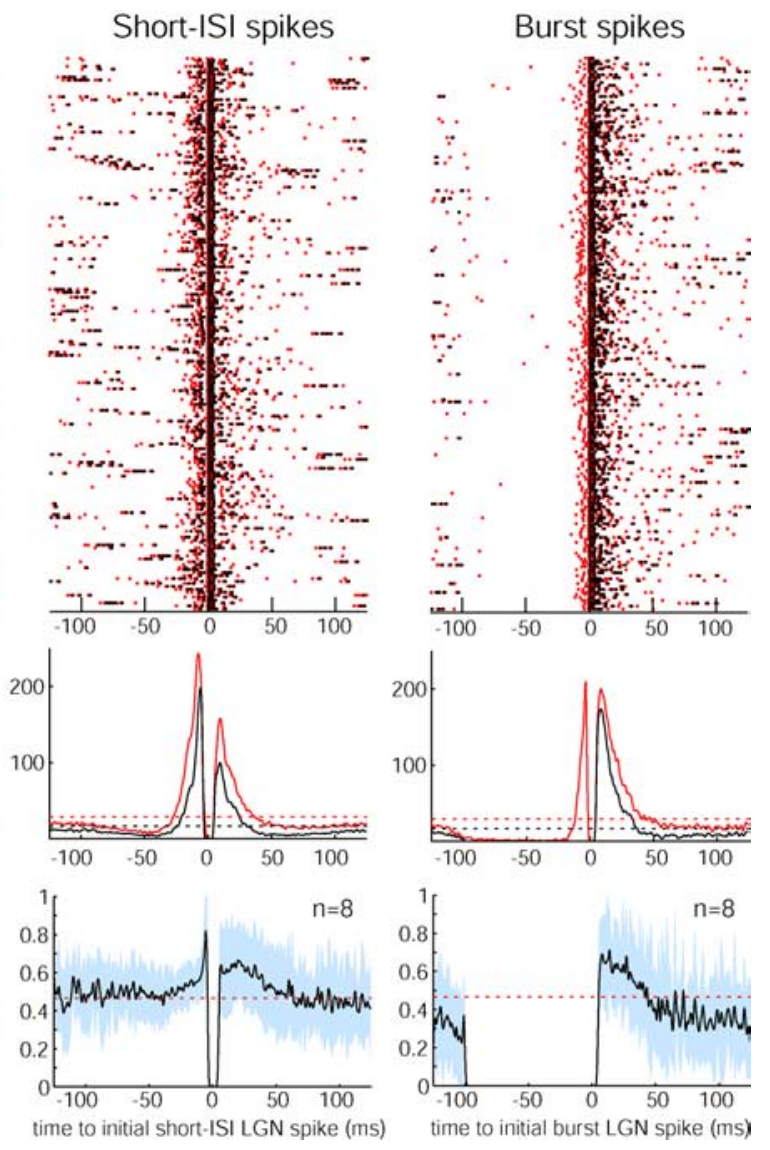

Figure 6. EPSP efficacy varies in time with LGN spike type. $\boldsymbol{A}, \mathrm{A}$ set of 200 rasters for each category of LGN spike from an 0 FF magnocellular neuron (LGN spike, black dot; EPSP, red dot). Rasters were centered at $t=0$ for the LGN tonic spikes and for the initial spikes in a short-ISI or burst spike train. $\boldsymbol{B}$, LGN spike auto-correlograms (black) and LGN-EPSP cross-correlograms (red) computed from the entire dataset for this cell (tonic, $n=13,267$; initial short-ISI, $n=3,560$; initial burst, $n=1519$ ). The correlograms were zeroed over the interval $-1<t<1 \mathrm{~ms}$ for tonic spikes and $-1<t<5 \mathrm{~ms}$ for short-ISI and burst spikes to avoid the large central peaks present by definition and were then smoothed with a 2-ms-wide Blackman filter. Bin size, $0.5 \mathrm{~ms}$. Dotted lines, Mean firing rates. C, Population EPSP efficacy profiles for each category of spike, centered on $t=0$ for a tonic LGN spike (left) or on the initial spike in a short-ISI or burst train (middle and right). For burst spikes, the efficacy was 0 by definition for $100 \mathrm{~ms}$ before the burst train. The number of short-ISI or burst spike trains was too small in seven cells to generate meaningful efficacy curves. Blue shading, One SD; red dotted line, mean efficacy.

integrated when dead times were $<50 \mathrm{~ms}$. At longer dead times, efficacy was enhanced only by the first EPSP in the pair. For instance, at an ISI of $10 \mathrm{~ms}$, mean second EPSP efficacy was 0.85 with a $15 \mathrm{~ms}$ dead time but dropped to 0.49 with a $75 \mathrm{~ms}$ dead time.

These data indicate that, as long as EPSPs occur within a period of $\sim 40 \mathrm{~ms}$, their impact on efficacy generally accumulates. With sufficiently short intervals, efficacy can approach 1 . Beyond $40 \mathrm{~ms}$, the signaling capability of any EPSP dissipates to nearly 0 . Together, the data show that short EPSP intervals are necessary for successful transmission. To quantify this point, we compiled a temporal histogram of the first EPSP that immediately preceded any successful EPSP (Fig. 7E). Successful and failed EPSPs had similar distributions before spikes, confirming the conclusion above that a transmitted EPSP has the same influence on subsequent EPSP efficacy as a failed EPSP. Overall, 98\% of all spikes were lead by an EPSP within $33 \mathrm{~ms}$. For retinogeniculate transmission to occur, therefore, EPSPs that are close in time must summate. The observed difference in firing rates and patterns between retinal ganglion cells and LGN neurons can be partially understood by the fact that an isolated EPSP rarely brings an LGN neuron to threshold. material).

\section{Discussion}

We found that most LGN neurons have one retinal ganglion cell input that accounts for nearly all LGN spikes sent to visual cortex. This idea is usually cast in terms of "contribution," defined as the percentage of LGN spikes that are caused by a given ganglion cell (Levick et al., 1972). In previous single-electrode studies, it has been difficult to determine how many ganglion cells drive an LGN cell (Hubel and Wiesel, 1961; Bishop et al., 1962; Lee et al., 1983; Kaplan and Shapley, 1984; Wang et al., 1985; Mastronarde, 1987). The EPSP is often merged with the LGN spike, making it appear that many LGN spikes have no associated EPSP. Through subtraction analysis, we have shown that this is not the case: $>95 \%$ of LGN spikes have an identifiable EPSP. For 92\% (34 of 37) of the LGN neurons, the EPSP had an absolute refractory period, signifying a $100 \%$ contribution. Thus, for the majority of LGN cells, the singleelectrode approach reveals a main ganglion cell driver.

A different story has emerged from dual-electrode recordings (Cleland et al., 1971; Levick et al., 1972; Cleland and Lee, 1985; Mastronarde, 1992; Usrey et al., 1999). With one electrode in the retina and another in the LGN, analysis of correlated firing has shown that LGN neurons are usually driven by more than one ganglion cell. Most ganglion cells have been found to provide a low contribution, generally <20\% (Mastronarde, 1987; Usrey et al., 1999). This low contribution is not surprising. Multiple retinal ganglion cells converge on a single LGN cell, but only one is the main driver. Therefore, the probability of encountering the main ganglion cell driver with a retinal electrode is low.

The results from single- and dual-electrode studies are reconcilable. Single-electrode recordings show that most LGN neurons have a main retinal input capable of initiating every spike, whereas dual-electrode recordings reveal that other ganglion cells provide additional, smaller inputs of low contribution. Therefore, the sum of the contributions from all retinal inputs is $>100 \%$, as reported by Cleland et al. (1971). The ganglion cells providing minor input apparently fail to produce EPSPs of sufficient amplitude to record extracellularly in the LGN. Their receptive field properties, however, are likely to match those of the primary driver (Cleland et al., 1971). Consequently, minor contributors are likely to fire in synchrony with the main retinal driver to generate LGN spikes. Infrequently, the EPSPs of a minor contributor are large enough to record extracellularly in the LGN. When this occurs, two different EPSPs are visible in the recording trace. Often they overlap, attesting to their synchronization (supplemental Fig. 2, available at www.jneurosci.org as supplemental

Anatomical studies indicate that there are $\sim 1.5-1.8$ million 
retinal ganglion cells (Perry and Cowey, 1985; Kim et al., 1996; Spear et al., 1996) and 1.4-1.8 million LGN neurons (Williams and Rakic, 1988; Blasco et al., 1999). The equal number of ganglion cells and LGN cells is compatible with the physiological result that most LGN neurons have one major retinal driver. The number of retinal ganglion cells that synapse onto a single LGN neuron is unknown. Reconstruction of a single optic tract fiber in the cat showed contacts on four geniculate cells, accounting for $100 \%$ of the input to one cell (Hamos et al., 1987). Unfortunately, analogous data are unavailable for the macaque, but it is possible that retinal afferents synapse with fewer geniculate cells in the macaque than in the cat.

Previous studies have documented that LGN cells can fire in two modes: tonic and burst (Steriade and Llinas, 1988; McCormick and Feeser, 1990; Sherman, 2001a; Llinas and Steriade, 2006). Timing criteria are used to differentiate these two modes in extracellular recordings. However, the appropriate criteria are uncertain, with both conservative ( $100 \mathrm{~ms}$ pause, $4 \mathrm{~ms}$ ISI) and liberal (50 ms pause, $6 \mathrm{~ms}$ ISI) standards ( $\mathrm{Lu}$ et al., 1992; Ramcharan et al., 2000a). Our ISI scatter plots did not show a distinct cluster for burst spikes (supplemental Fig. 3, available at www.jneurosci.org as supplemental material), which suggests that timing criteria do not cleanly separate burst spikes from tonic spikes. Intracellular recordings have shown that burst spikes are associated with activation of $I_{\mathrm{T}}$ current and that prolonged hyperpolarization causes deinactivation of $I_{\mathrm{T}}$ (Lu et al., 1992). Consequently, a single EPSP should be sufficient to activate $I_{\mathrm{T}}$ current, thereby generating several spikes without additional retinal input (Jahnsen and Llinas, 1984; Crunelli et al., 1989; Lu et al., 1992; Zhan et al., 1999; Ramcharan et al., 2000b). Based on these studies, we expected to find no EPSPs associated with the secondary spikes in the bursts in our recordings. However, we found that nearly all spikes in a burst had identifiable retinal EPSPs. Thus, under our experimental conditions, LGN bursts seemed to originate from retinal bursts. It was uncertain whether these bursts were accompanied by $I_{\mathrm{T}}$-driven membrane depolarization because we recorded extracellularly. During visual stimulation, it is possible that LGN neurons do not sustain the hyperpolarization of less than $-70 \mathrm{mV}$ that is needed to activate $I_{\mathrm{T}}$, as reported in vitro (Alexander et al., 2006). A timing criterion alone is insufficient for identifying $I_{\mathrm{T}}$-driven bursts in the LGN.

Given that EPSPs were associated with nearly every LGN burst spike, one should be able to record burst activity in retinal ganglion cells. In a previous study, burst activity was reported to be absent in optic tract units (Lo et al., 1991). This observation lent support to the idea that burst activity is generated by $I_{\mathrm{T}}$ current in LGN cells. These optic tract recordings, however, were made in the absence of visual stimulation. Under conditions of naturalistic stimulation, our optic tract recordings showed that burst firing does occur in retinal ganglion cells. Interestingly, T-type $\mathrm{Ca}^{2+}$ channels have been identified in goldfish, salamander, rat, and cat retina, providing a potential substrate for burst firing (Karschin and Lipton, 1989; Huang and Robinson, 1998; Henderson and Miller, 2003; Lee et al., 2003). Their presence in macaque ganglion cells is unknown.

Bursts in the LGN were originally found to occur when an animal is asleep or drowsy (Hubel, 1960; Livingstone and Hubel, 1981; McCarley et al., 1983). During sleep, rhythmic bursting involves repeated cycles of $I_{\mathrm{T}}$ current activation and inactivation (McCormick and Feeser, 1990; Steriade et al., 1993). Tonic firing prevails when an animal is alert but bursts also occur, albeit at a relatively low frequency (Guido and Weyand, 1995; Ramcharan et al., 2000a; Weyand et al., 2001; Ruiz et al., 2006). No study has 
compared the prevalence of bursts in alert, sleep, and anesthetized states. The percentage of burst spikes (mean of 3.7\%) we observed in anesthetized animals was comparable with the low percentage reported in alert animals (Hubel, 1960; Livingstone and Hubel, 1981; McCarley et al., 1983; Guido and Weyand, 1995; Ramcharan et al., 2000a, 2005; Weyand et al., 2001; Ruiz et al., 2006). Thus, our experimental conditions did not produce an unusually low burst rate.

It has been observed that thalamic bursts are indicative of a sleep state (Hirsch et al., 1983; Steriade et al., 1993; Sherman, 2001b; Llinas and Steriade, 2006). The electroencephalograms in our animals showed constant low-amplitude $\beta$ activity during experiments, without the typical features of sleep. Therefore, the bursts that we observed were not related to transitions between sleep and wakefulness. Another potential role for bursts is to signal salient or novel features in the visual environment (Guido et al., 1995; Reinagel et al., 1999; Lesica and Stanley, 2004; Alitto et al., 2005; Denning and Reinagel, 2005). This concept is appealing because quiescent LGN cells are likely to be transiently activated by such stimuli. However, the same stimuli can also transiently activate ganglion cells, resulting in LGN bursts that originate in the retina. Stimulus novelty can still be signaled by bursts but now must be occasionally attributed to retinal processing.

We found that EPSP efficacy was dependent on the interval between EPSPs during naturalistic stimulation. Previous studies using the dual-electrode approach and white noise or grating stimuli also found paired retinal spike facilitation (Mastronarde, 1987; Usrey et al., 1998, 1999; Rowe and Fischer, 2001). In these studies, mean efficacy (usually $<15 \%$ ) and contribution (usually $<20 \%$ ) were low. Nonetheless, postspike facilitation lasted 40 $\mathrm{ms}$, similar to the time course of efficacy enhancement we observed. This finding suggests that the temporal properties of the EPSP integration mechanism are not sensitive to differences in mean EPSP efficacy or ganglion cell contribution.

We found that EPSP efficacy was not influenced by the occurrence of a spike after the first EPSP in a pair. Action potentials initiated at the axon initial segment propagate back into the dendrites of LGN neurons (Williams and Stuart, 2000). In Purkinje cells and in cortical layer 5 pyramidal cells, it has been shown that spikes shunt EPSPs to a variable degree in vitro (Hausser et al., 2001). Shunting increases the synaptic current required to generate a spike after the next EPSP. Therefore, we expected that second EPSP efficacy would decrease after conversion of an EPSP into a spike, but this was not observed for any retinal ISI (Fig. 7C). The absence of any shunting effect suggests that backpropagation of action potentials (the somatodendritic potentials we recorded) does not perturb the integration of EPSPs. The facilitation of EPSPs may be attributable to NMDA current, which is less affected by spike shunting because of slower kinetics (Hausser et al., 2001). Indeed, during physiologically realistic retinal spike trains, each input produces sizable AMPA and NMDA currents, with the latter capable of summing at high frequencies (Chen et al., 2002; Blitz and Regehr, 2003). This summation of NMDA current probably helps to achieve the high efficacy rates observed within a rapid spike train, as shown by the dependence of sustained responses on NMDA current in vivo (Hartveit and Heggelund, 1990; Sillito et al., 1990; Kwon et al., 1991).

After a quiescent period, LGN neurons did not spike to the first EPSP to come along. Bursts, for instance, needed at least one failed EPSP before subsequent EPSPs were transduced. The requirement for EPSP summation before LGN neurons began spiking was found for all spike types, not just for burst trains. Failed
EPSPs that are followed by successful EPSPs account for a substantial fraction of the retinal spike train that has been often considered lost in retinogeniculate transmission. These inputs are not wasted, however, because they presumably raise the membrane potential so that the next EPSPs achieve reliable transmission. With $98 \%$ of "priming" EPSPs falling within $33 \mathrm{~ms}$ of a spike, it appears that an instantaneous firing rate of $30 \mathrm{~Hz}$ is needed to bring an LGN neuron to threshold. The summation requirement explains the threshold portion of the function that relates the retinal spike rate to LGN spike rate (Lee et al., 1983; Movshon et al., 2005). This threshold nonlinearity is incorporated as the final stage of most neural models relating synaptic input to spike output (Dayan and Abbott, 2001).

\section{References}

Alexander GM, Carden WB, Mu J, Kurukulasuriya NC, McCool BA, Nordskog BK, Friedman DP, Daunais JB, Grant KA, Godwin DW (2006) The native T-type calcium current in relay neurons of the primate thalamus. Neuroscience 141:453-461.

Alitto HJ, Weyand TG, Usrey WM (2005) Distinct properties of stimulusevoked bursts in the lateral geniculate nucleus. J Neurosci 25:514-523.

Bishop PO, Burke W, Davis R (1958) Synapse discharge by single fibre in mammalian visual system. Nature 182:728-730.

Bishop PO, Burke W, Davis R (1962) The interpretation of the extracellular response of single lateral geniculate cells. J Physiol (Lond) 162:451-472.

Blasco B, Avendano C, Cavada C (1999) A stereological analysis of the lateral geniculate nucleus in adult Macaca nemestrina monkeys. Vis Neurosci 16:933-941.

Blitz DM, Regehr WG (2003) Retinogeniculate synaptic properties controlling spike number and timing in relay neurons. J Neurophysiol 90:2438-2450.

Chen C, Blitz DM, Regehr WG (2002) Contributions of receptor desensitization and saturation to plasticity at the retinogeniculate synapse. Neuron 33:779-788.

Cleland BG, Lee BB (1985) A comparison of visual responses of cat lateral geniculate nucleus neurones with those of ganglion cells afferent to them. J Physiol (Lond) 369:249-268.

Cleland BG, Dubin MW, Levick WR (1971) Simultaneous recording of input and output of lateral geniculate neurones. Nat New Biol 231:191-192.

Crunelli V, Lightowler S, Pollard CE (1989) A T-type $\mathrm{Ca}^{2+}$ current underlies low-threshold $\mathrm{Ca}^{2+}$ potentials in cells of the cat and rat lateral geniculate nucleus. J Physiol (Lond) 413:543-561.

Dan Y, Atick JJ, Reid RC (1996) Efficient coding of natural scenes in the lateral geniculate nucleus: experimental test of a computational theory. J Neurosci 16:3351-3362.

Dayan P, Abbott LF (2001) Theoretical neuroscience: computational and mathematical modeling of neural systems. Cambridge, MA: MIT.

Denning KS, Reinagel P (2005) Visual control of burst priming in the anesthetized lateral geniculate nucleus. J Neurosci 25:3531-3538.

Derrington AM, Lennie P (1984) Spatial and temporal contrast sensitivities of neurones in lateral geniculate nucleus of macaque. J Physiol (Lond) 357:219-240.

Guido W, Weyand T (1995) Burst responses in thalamic relay cells of the awake behaving cat. J Neurophysiol 74:1782-1786.

Guido W, Lu SM, Vaughan JW, Godwin DW, Sherman SM (1995) Receiver operating characteristic (ROC) analysis of neurons in the cat's lateral geniculate nucleus during tonic and burst response mode. Vis Neurosci 12:723-741.

Hamos JE, Van Horn SC, Raczkowski D, Sherman SM (1987) Synaptic circuits involving an individual retinogeniculate axon in the cat. J Comp Neurol 259:165-192.

Hartveit E, Heggelund P (1990) Neurotransmitter receptors mediating excitatory input to cells in the cat lateral geniculate nucleus. II. Nonlagged cells. J Neurophysiol 63:1361-1372.

Hausser M, Major G, Stuart GJ (2001) Differential shunting of EPSPs by action potentials. Science 291:138-141.

Henderson D, Miller RF (2003) Evidence for low-voltage-activated (LVA) calcium currents in the dendrites of tiger salamander retinal ganglion cells. Vis Neurosci 20:141-152.

Hirsch JC, Fourment A, Marc ME (1983) Sleep-related variations of mem- 
brane potential in the lateral geniculate body relay neurons of the cat. Brain Res 259:308-312.

Huang SJ, Robinson DW (1998) Activation and inactivation properties of voltage-gated calcium currents in developing cat retinal ganglion cells. Neuroscience 85:239-247.

Hubel DH (1960) Single unit activity in lateral geniculate body and optic tract of unrestrained cats. J Physiol (Lond) 150:91-104.

Hubel DH, Wiesel TN (1961) Integrative action in the cat's lateral geniculate body. J Physiol (Lond) 155:385-398.

Jahnsen H, Llinas R (1984) Ionic basis for the electro-responsiveness and oscillatory properties of guinea-pig thalamic neurones in vitro. J Physiol (Lond) 349:227-247.

Kaplan E, Shapley R (1984) The origin of the S (slow) potential in the mammalian lateral geniculate nucleus. Exp Brain Res 55:111-116.

Kaplan E, Purpura K, Shapley RM (1987) Contrast affects the transmission of visual information through the mammalian lateral geniculate nucleus. J Physiol (Lond) 391:267-288.

Karschin A, Lipton SA (1989) Calcium channels in solitary retinal ganglion cells from post-natal rat. J Physiol (Lond) 418:379-396.

Kim CB, Tom BW, Spear PD (1996) Effects of aging on the densities, numbers, and sizes of retinal ganglion cells in rhesus monkey. Neurobiol Aging 17:431-438.

Kwon YH, Esguerra M, Sur M (1991) NMDA and non-NMDA receptors mediate visual responses of neurons in the cat's lateral geniculate nucleus. J Neurophysiol 66:414-428.

Lee BB, Virsu V, Creutzfeldt OD (1983) Linear signal transmission from prepotentials to cells in the macaque lateral geniculate nucleus. Exp Brain Res 52:50-56.

Lee SC, Hayashida Y, Ishida AT (2003) Availability of low-threshold $\mathrm{Ca}^{2+}$ current in retinal ganglion cells. J Neurophysiol 90:3888-3901.

Lesica NA, Stanley GB (2004) Encoding of natural scene movies by tonic and burst spikes in the lateral geniculate nucleus. J Neurosci 24:10731-10740.

Levick WR, Cleland BG, Dubin MW (1972) Lateral geniculate neurons of cat: retinal inputs and physiology. Invest Ophthalmol 11:302-311.

Levitt JB, Schumer RA, Sherman SM, Spear PD, Movshon JA (2001) Visual response properties of neurons in the LGN of normally reared and visually deprived macaque monkeys. J Neurophysiol 85:2111-2129.

Lewicki MS (1998) A review of methods for spike sorting: the detection and classification of neural action potentials. Network 9:R53-R78.

Livingstone MS, Hubel DH (1981) Effects of sleep and arousal on the processing of visual information in the cat. Nature 291:554-561.

Llinas RR, Steriade M (2006) Bursting of thalamic neurons and states of vigilance. J Neurophysiol 95:3297-3308.

Lo FS, Lu SM, Sherman SM (1991) Intracellular and extracellular in vivo recording of different response modes for relay cells of the cat's lateral geniculate nucleus. Exp Brain Res 83:317-328.

Lu SM, Guido W, Sherman SM (1992) Effects of membrane voltage on receptive field properties of lateral geniculate neurons in the cat: contributions of the low-threshold $\mathrm{Ca}^{2+}$ conductance. J Neurophysiol 68:2185-2198.

Mastronarde DN (1987) Two classes of single-input X-cells in cat lateral geniculate nucleus. II. Retinal inputs and the generation of receptive-field properties. J Neurophysiol 57:381-413.

Mastronarde DN (1992) Nonlagged relay cells and interneurons in the cat lateral geniculate nucleus: receptive-field properties and retinal inputs. Vis Neurosci 8:407-441.

McCarley RW, Benoit O, Barrionuevo G (1983) Lateral geniculate nucleus unitary discharge in sleep and waking: state- and rate-specific aspects. J Neurophysiol 50:798-818.

McCormick DA, Feeser HR (1990) Functional implications of burst firing and single spike activity in lateral geniculate relay neurons. Neuroscience 39:103-113.

Movshon JA, Kiorpes L, Hawken MJ, Cavanaugh JR (2005) Functional maturation of the macaque's lateral geniculate nucleus. J Neurosci 25:2712-2722.

Perry VH, Cowey A (1985) The ganglion cell and cone distributions in the monkey's retina: implications for central magnification factors. Vision Res 25:1795-1810.

Ramcharan EJ, Gnadt JW, Sherman SM (2000a) Burst and tonic firing in thalamic cells of unanesthetized, behaving monkeys. Vis Neurosci 17:55-62.

Ramcharan EJ, Cox CL, Zhan XJ, Sherman SM, Gnadt JW (2000b) Cellular mechanisms underlying activity patterns in the monkey thalamus during visual behavior. J Neurophysiol 84:1982-1987.

Ramcharan EJ, Gnadt JW, Sherman SM (2005) Higher-order thalamic relays burst more than first-order relays. Proc Natl Acad Sci USA 102:12236-12241.

Reinagel P, Reid RC (2000) Temporal coding of visual information in the thalamus. J Neurosci 20:5392-5400.

Reinagel P, Godwin D, Sherman SM, Koch C (1999) Encoding of visual information by LGN bursts. J Neurophysiol 81:2558-2569.

Rowe MH, Fischer Q (2001) Dynamic properties of retino-geniculate synapses in the cat. Vis Neurosci 18:219-231.

Ruiz O, Royal D, Sary G, Chen X, Schall JD, Casagrande VA (2006) Lowthreshold $\mathrm{Ca}^{2+}$-associated bursts are rare events in the LGN of the awake behaving monkey. J Neurophysiol 95:3401-3413.

Sherman SM (2001a) Thalamic relay functions. Prog Brain Res 134:51-69.

Sherman SM (2001b) Tonic and burst firing: dual modes of thalamocortical relay. Trends Neurosci 24:122-126.

Sillito AM, Murphy PC, Salt TE, Moody CI (1990) Dependence of retinogeniculate transmission in cat on NMDA receptors. J Neurophysiol 63:347-355.

Spear PD, Kim CB, Ahmad A, Tom BW (1996) Relationship between numbers of retinal ganglion cells and lateral geniculate neurons in the rhesus monkey. Vis Neurosci 13:199-203.

Steriade M, Llinas RR (1988) The functional states of the thalamus and the associated neuronal interplay. Physiol Rev 68:649-742.

Steriade M, McCormick DA, Sejnowski TJ (1993) Thalamocortical oscillations in the sleeping and aroused brain. Science 262:679-685.

Swanson WH, Ueno T, Smith VC, Pokorny J (1987) Temporal modulation sensitivity and pulse-detection thresholds for chromatic and luminance perturbations. J Opt Soc Am A 4:1992-2005.

Usrey WM, Reppas JB, Reid RC (1998) Paired-spike interactions and synaptic efficacy of retinal inputs to the thalamus. Nature 395:384-387.

Usrey WM, Reppas JB, Reid RC (1999) Specificity and strength of retinogeniculate connections. J Neurophysiol 82:3527-3540.

van Hateren JH (1997) Processing of natural time series of intensities by the visual system of the blowfly. Vision Res 37:3407-3416.

Wang C, Cleland BG, Burke W (1985) Synaptic delay in the lateral geniculate nucleus of the cat. Brain Res 343:236-245.

Weyand TG, Boudreaux M, Guido W (2001) Burst and tonic response modes in thalamic neurons during sleep and wakefulness. J Neurophysiol $85: 1107-1118$.

Williams RW, Rakic P (1988) Elimination of neurons from the rhesus monkey's lateral geniculate nucleus during development. J Comp Neurol 272:424-436.

Williams SR, Stuart GJ (2000) Action potential backpropagation and somato-dendritic distribution of ion channels in thalamocortical neurons. J Neurosci 20:1307-1317.

Zhan XJ, Cox CL, Rinzel J, Sherman SM (1999) Current clamp and modeling studies of low-threshold calcium spikes in cells of the cat's lateral geniculate nucleus. J Neurophysiol 81:2360-2373. 